\section{W. Tilgen \\ K. Rass \\ J. Reichrath}

\title{
30 Jahre dermatologische Onkologie
}

\section{Years of Dermato-Oncology}

\section{Zusammenfassung}

Basierend auf einer stetig gewachsenen Kompetenz im Bereich der Tumortherapie und -diagnostik hat sich die Dermato-Onkologie in den letzten Jahrzehnten so rasant entwickelt wie kaum ein anderes Teilgebiet der Dermatologie. Veränderte Lebensgewohnheiten und die zunehmende Lebenserwartung belasten das Grenzorgan „Haut“ durch Exposition gegenüber unterschiedlichen Karzinogenen, wie UV-Licht, in besonderem Maße. Dies spiegelt sich in steigenden Inzidenzraten für epitheliale Tumore, die mittlerweile die höchste Inzidenz aller bösartigen Tumoren aufweisen, und das Melanom wieder. Dieser Herausforderung begegneten die deutschen Dermatologen durch konzertierte Aktionen zur primären und sekundären Prävention. Regelmäßig aktualisierte Leitlinien zu allen relevanten Hauttumoren gewährleisten heute zudem ein hohes Maß an Qualitätssicherung und reflektieren die Kompetenz unseres Fachgebietes. Als Ausdruck des hohen Stellenwertes einer evidenzbasierten Medizin stellen multizentrische Therapiestudien in der Dermato-Onkologie heute den Standard in der Behandlung zahlreicher Tumorentitäten dar. Beeindruckende Fortschritte wurden auch auf dem Gebiet der dermato-onkologischen Grundlagenforschung erzielt. Das Wissen über die Entstehung und das Metastasierungsverhalten der Hauttumore hat sich durch Erkenntnisse in der Molekularbiologie und Immunologie entscheidend erweitert und neuen Therapieansätzen den Weg gebahnt. Aus dem breiten Spektrum maligner Hauttumoren wird die Entwicklung „30 Jahre DermatoOnkologie“ am Beispiel des Basalzell-, des Plattenepithelkarzinoms, des Melanoms und kutaner Lymphome aufgezeigt.

\section{Abstract}

Based on a steadily increasing expertise in tumor therapy and diagnosis, dermato-oncology has been fast paced as almost no other section of dermatology. Changes in habits and increased life expectancy burden the "frontier organ skin" to an increasing degree with exposition against various carcinogens, including UV-light. This is reflected by increasing incidence rates for epithelial tumors, that in the meantime exhibit the highest incidence of all malignancies, and for melanoma. This challenge has been encountered by dermatologists by activities for primary and secondary prevention. Continuously updated guidelines for all relevant skin tumors guarantee a high degree of quality assurance and reflect our competence. Today, multicenter studies represent the standard in the treatment of multiple malignancies, reflecting the importance of evidence-based medicine. Impressing progress has also been achieved in basic research in dermatooncology. Our knowledge about pathogenesis and progression of skin tumors has been critically increased by new insights in molecular biology and immunology, thus paving the way for innovative therapy approaches. Out of the wide spectrum of malignant skin tumors, we here report the development of "30 Years Dermato-Oncology", at the example of melanoma, cutaneous lymphomas, basal and squamous cell carcinomas. 
Kaum ein Teilgebiet der Dermatologie hat sich in den letzten Jahrzehnten so rasant entwickelt wie die Dermato-Onkologie. Es fand eine weitreichende Umorientierung unseres Fachgebietes und gleichzeitige Loslösung aus der Inneren Medizin und Chirurgie statt. Diese Selbstständigkeit basiert insbesondere auf der stetig gewachsenen Kompetenz im Bereich der Tumortherapie und -diagnostik (Tab. 1 [1]).

Veränderte Lebensgewohnheiten und die zunehmende Lebenserwartung belasten das Grenzorgan „Haut“ durch Exposition gegenüber unterschiedlichen Karzinogenen, wie UV-Licht, in besonderem Maße. Dies spiegelt sich in steigenden Inzidenzraten für epitheliale Tumore, die mittlerweile die höchste Inzidenz aller bösartigen Tumoren aufweisen, und das Melanom wider. In Deutschland erkranken rund 120000 Menschen jedes Jahr neu an Hautkrebs, etwa 3000 sterben daran. Etwa 11400 Menschen erkranken jährlich an einem malignen Melanom. Dieser Herausforderung begegneten die deutschen Dermatologen durch konzertierte Aktionen zur primären und sekundären Prävention. Die Einsicht, dass nur gemeinsame Anstrengungen zu verlässlichen Aussagen führen, fand ihren Niederschlag in dem größten Melanomregister der Welt und in der Gründung mehrerer Arbeitsgemeinschaften.

Besonders beeindruckende Fortschritte wurden auf dem Gebiet der dermato-onkologischen Grundlagenforschung erzielt. Das Wissen über die Entstehung und das Metastasierungsverhalten der Hauttumore hat sich durch Erkenntnisse in der Molekularbiologie und Immunologie entscheidend erweitert.

Die rasche Entwicklung moderner Biotechnologien hat zahlreiche neue Ansätze für Diagnostik und Therapie ermöglicht, von denen allerdings nur ein kleiner Teil über das experimentelle Stadium hinaus Einzug in die klinische Praxis gehalten hat. Bemerkenswert in der Therapie ist ein Paradigmenwechsel: Nicht mehr die Ansprechrate eines Tumors auf eine bestimmte Therapie steht im Vordergrund des Interesses, sondern das Überleben unter Berücksichtigung der Lebensqualität. Insgesamt ist die Radikalität des Vorgehens einer stadiengerechten Diagnostik und Therapie gewichen.

Als Ausdruck des hohen Stellenwertes einer evidenzbasierten Medizin stellen multizentrische Therapiestudien heute den Standard in der Behandlung zahlreicher Tumorentitäten dar. Ergebnis interdisziplinärer Konsensfähigkeit sind regelmäßig aktualisierte Leitlinien zu allen relevanten Hauttumoren [2]. Diese gewährleisten ein hohes Maß an Qualitätssicherung und reflektieren die Kompetenz unseres Fachgebietes.

Insbesondere beim Melanom führten die aus den Studien und dem Melanomregister gewonnenen Erkenntnisse zu neuen Konzepten in der Nachsorge, die eine wesentliche Erleichterung für den Patienten bedeuten.

Der technologische Innovationsschub und die Möglichkeit molekularbiologische Prozesse in der Tumorzelle zu beeinflussen führten allerdings auch zu neuen ethischen Herausforderungen. Hinzu kommen die Zwänge einer Ökonomisierung in der Medi-
Tab. 1 Herausragende Entwicklung der Dermatologie auf dem Gebiet der Dermato-Onkologie

\section{Gründung von Arbeitsgemeinschaften Gesellschaften}

Vereinigung für operative Dermatologie (1977), seit 1989 Vereinigung für operative und onkologische Dermatologie

Arbeitsgemeinschaft Dermatologische Onkologie (1990), hervorgegangen aus der „Kommission malignes Melanom“ der Deutschen Dermatologischen Gesellschaft (Beginn der 80er-Jahre)

Kommission zur Früherkennung und Prävention von Hautkrebs (1987)

Arbeitsgemeinschaft Dermatologische Prävention (1994)

Mitgliedschaften in nationalen und internationalen Gesellschaften EORTC

EADO (Gründung und Mitgliedschaft seit 1998)

Deutsche Krebsgesellschaft (ADO-Mitgliedschaft seit 1994)

Bundesministerium für Gesundheit und Soziales (BMGS), Kommission Prävention und Epidemiologie (1989)

Bundesministerium für Umwelt (BMU), Strahlenschutzkommission (1991)

Unterausschuss Prävention des Gemeinsamen Bundesausschuss (GBA) (1996) International Commission of Non-ionising Radiation Protection (ICNIRP) (2004)

Gründung Deutsche Hautkrebsstiftung (ca. 1998)

Etablierung Melanomregister (1983)

(weltweit das größte Melanomregister mit über 60000 Patienten)

Gründung Zentralregister kutane Laymphome der DDG (1998)

Erstellung von Leitlinien $(\mathbf{1 9 9 8 , 2 0 0 4 )}[4,5,13,33]$

Herausgeberschaft von Empfehlungen für eine standardisierte Diagnostik, Therapie und Nachsorge maligner Melanome im Auftrag deutscher Tumorzentren

Herausgeberschaft von Büchern zum Thema Dermato-Onkologie

(Auswahl)

Fortschritte der operativen Dermatologie/Fortschritte der operativen und onkologischen Dermatologie (Jahrestagungen der Vereinigung für operative und onkologische Dermatologie, seit 1978, 26 Jahrestagungen, 19 Kongressbände)

Das Basaliom: Der häufigste Tumor der Haut (Hrsg. F. Eichmann, U. W. Schnyder, 1981)

Das maligne Melanom der Haut (F. Weidner, J. Tonak, 1981)

Cutaneous lymphomas, pseudolymphomas and related disorders (G. Burg, O. Braun-Falco, 1983)

Onkologie der Haut (Hrsg. J. Petres, J. Kunze, R. P. A Müller, 1984)

Kutane Melanome: Klinik und Differenzialdiagnose (R. Kaufmann, L. Weber, O. E. Rodermund, 1989)

Das maligne Melanom der Haut (Hrsg. C. E. Orfanos, C. Garbe, 1990)

Malignant Melanoma: Diagnosis and Differential Diagnosis (E. Hölzle, P. Kind, G. Plewig, W. Burgdorf. Schattauer, 1993)

Das Basaliom. Klinik und Therapie (Hrsg. J. Petres, I. Lohrisch, 1993)

Recent Results in Cancer Research. Skin carcinogenesis in man and in experimental models (Hrsg. E. Hecker, E. G. Jung, F. Marks, W. Tilgen, 1993)

Malignes Melanom: Therapiestrategien und Nachsorgekonzepte (Hrsg. W. Meigel, 1993)

Recent Results in Cancer Research. Skin cancer. Basic science, clinical research and treatment (Hrsg. C. Garbe, S. Schmitz, C. E. Orfanos, 1995)

Dermatologische Onkologie (Hrsg. C. Garbe, R. Dummer, R. Kaufmann, W. Tilgen, 1997)

Klinik des metastasierten malignen Melanoms. Grundlagen, Diagnostik, Therapie (Hrsg. H. D. Böttcher, R. Kaufmann, 1999)

Das maligne Melanom der Haut (G. Sebastian, A. Stein, 2000)

Recent Results in Cancer Research. Minimal residual disease in melanoma. Biology, detection and clinical relevance (Hrsg. U. Reinhold, W. Tilgen, 2000)

Recent Results in Cancer Research. Chemosensitivity testing in oncology (Hrsg. U. Reinhold, W. Tilgen, 2003)

Recent Results in Cancer Research. Vitamin D analogs in cancer prevention and therapy (Hrsg. J. Reichrath, M. Friedrich, W. Tilgen, 2003)

Herausgeberschaften von Themenheften zu Dermato-Onkologie (z. B. „Der Onkologe“, „Onkologie“)

Ehrungen auf dem Gebiet der Dermato-Onkologie

Verleihung des Deutschen Krebspreises: Garbe 1995, Dummer 2003, Becker 2004

Verleihung des Deutschen Hautkrebspreises (seit 2001): Becker, Friedl, Hauschild, Reinhold, Schuler-Thurner, Startz

Verleihung des Ernst-von-Leyden-Preises des Deutschen

Krebsforschungszentrum (DKFZ): Breitbart 1990 


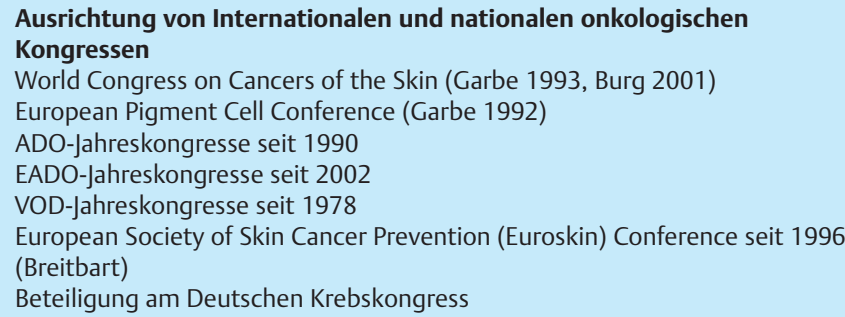

Die Tabelle erhebt keinen Anspruch auf Vollständigkeit.

zin, u.a. durch die Auswirkungen des Gesundheitsmodernisierungsgesetzes, durch die die Forderung „Im Mittelpunkt steht der Mensch“ ins Wanken und in eine Schieflage zu geraten droht.

Die gesamte Spannweite neuerer Erkenntnisse in der DermatoOnkologie kann im Rahmen dieses Rückblicks nicht wiedergegeben werden (z. B. der Nachweis von HHV-8 beim Kaposi-Sarkom, die Entdeckung einer spezifischen chromosomalen Translokation beim Dermatofibrosarkoma protuberans, der Nachweis von Somatostatinrezeptoren beim Merkelzellkarzinom und den daraus resultierenden Therapieansätzen).

Aus dem breiten Spektrum maligner Hauttumoren soll die Entwicklung „30 Jahre Dermato-Onkologie“ am Beispiel des Basalzell-, des Plattenepithelkarzinoms, des Melanoms und kutaner Lymphome aufgezeigt werden.

\section{Jahre Dermato-Onkologie: Epitheliale Hauttumoren (Basalzellkarzinome, Plattenepithelkarzinome)}

Epidemiologie: Die Inzidenz von epithelialen Hauttumoren hat in den letzten 30 Jahren erheblich zugenommen. Zur Zeit beträgt sie in Europa für Basalzellkarzinome bis zu $0,1-0,2 \%$ und für Plattenepithelkarzinome bis zu 0,03\% [3-5]. Für die Entwicklung epithelialer Tumore sind genetische und Umweltfaktoren in gleicher Weise von Bedeutung. Eine genetische Disposition zur Tumorentstehung haben hellhäutige Menschen mit einer hohen Lichtempfindlichkeit. Einen wesentlichen Risikofaktor stellt die individuelle kumulative UV-Exposition dar. Während einige der klassischen Risikofaktoren, wie beispielsweise die Arsenexposition, heute für die Verursachung von Basalzell- und Plattenepithelkarzinomen keine wesentliche Bedeutung mehr haben, besteht ein erhöhtes Risiko Hauttumoren zu entwickeln bei immunsupprimierten Patienten. Diese Problematik hat in den letzten Jahren u.a. durch die stark zunehmende Zahl von Organtransplantationen an Bedeutung gewonnen. Weitere wesentliche Faktoren für die Zunahme der Inzidenz von Hautkrebs sind die geänderte Alterspyramide und das veränderte Freizeitverhalten, welche eine erhöhte individuelle UV-Exposition bewirken (Fernreisen, Schönheitsideal gebräunte Haut; UV-Exposition: „früher - mehr - länger“) [6]. Zudem wird die Intensität der UVBelastung an der Erdoberfläche in den letzten 30 Jahren durch die erhebliche Zunahme des Ozonlochs deutlich verstärkt. Die Ozonschicht wurde in den letzten 30 Jahren um ca. 10\% ge- schwächt und wird sich voraussichtlich jedes kommende Jahr um weitere $0,5 \%$ verringern. Eine einprozentige Ozonabnahme bedeutet eine 1,5-2\%ige Zunahme der UV-B-Intensität an der Erdoberfläche, dies führt nach Schätzungen in unseren Breiten zu einer Erhöhung der Hautkrebsrate von etwa 3\% [7]

Ätiologie: Unser Verständnis über die Pathogenese epithelialer Hauttumoren hat sich in den letzten 30 Jahren wesentlich verbessert (Tab.2). Wie für andere Malignome wurde auch für Hauttumoren ein Mehrschrittmodell der Karzinogenese nachgewiesen. Wichtigstes Karzinogen ist das UV-Licht, welches eine so genannte „Field cancerization“ bewirkt. UV-Licht-bedingte DNS-Schädigungen legen im Rahmen der Tumor-Initiation danach den Grundstein für eine spätere Tumorentwicklung. In der zweiten Phase, der Promotion, und in der dritten Phase, der Tumorprogression, spielt die UV-Bestrahlung ebenfalls eine wichtige Rolle [8]. Eine exzessive Sonnenexposition ist daher der wichtigste Umweltfaktor im Rahmen der Photokarzinogenese. Die UV-B-Strahlung bewirkt eine Dosis-abhängige direkte Schädigung der DNS, was strukturelle Veränderungen (z.B. Cyclobutyldimere, Pyrimidin-6-4-Verbindungen, Einzelstrangbrüche und DNS-Protein-Vernetzungen) initiiert, die wiederum zu Deletionen während der Replikation führen können $[8,9]$.

Neben dem natürlichen Lichtschutz der Haut, der auf einer UVStrahlen-induzierten Verbreiterung der Epidermis - der so genannten Lichtschwiele - und einer Vermehrung von Melanin in den Keratinozyten beruht, hat man in den letzten Jahren neue Erkenntnisse über hauteigene DNS-Reparaturmechanismen gewonnen. Zwei DNS-Reparaturmechanismen sind entscheidend: Die Exzisionsreparatur: Der geschädigte DNS-Abschnitt wird durch einen „Dark-repair“-Mechanismus selektiv entfernt und durch passende Nukleotide ersetzt. Die DNS-Funktion ist wiederherge-

Tab. 2 Entwicklungen in der Dermato-Onkologie: Basalzell- und Plattenepithelkarzinome

\begin{tabular}{|c|c|c|}
\hline & 1974 & heute \\
\hline Inzidenz & $?$ & $\begin{array}{l}\text { BCC: ca. } 0,1-0,2 \% \\
\text { PEC: ca. } 0,03 \%\end{array}$ \\
\hline Ätiologie & $?$ & $\begin{array}{l}\text { PEC: p53, } \\
\text { onkogene Viren } \\
\text { DNS-Reparatursystem } \\
\text { BCC: PATCHED/HEDGEHOG } \\
\text { DNS-Reparatursystem }\end{array}$ \\
\hline Risikofaktoren & Arsen, UV & $\begin{array}{l}\text { UV (Alterspyramide, } \\
\text { Schönheitsideal, gebräunte } \\
\text { Haut,Freizeitverhalten, } \\
\text { Fernreisen) }\end{array}$ \\
\hline Prävention & Sonnenschutz (UV-B) & $\begin{array}{l}\text { umfassende Präventions- } \\
\text { programme zum Sonnen- } \\
\text { schutz (UV-A/UV-B) } \\
\text { Photolyase } \\
\text { Retinoide? }\end{array}$ \\
\hline Therapie & $\begin{array}{l}\text { Chirurgisch (Mikrochirurgie } \\
\text { nach Moh) } \\
\text { Dermopan } \\
\text { Zytostatika }\end{array}$ & $\begin{array}{l}\text { Chirurgisch (Mikrochirurgie } \\
\text { nach Moh, Breuninger) } \\
\text { (Dermopan) } \\
\text { Photodynamische Therapie } \\
\text { Immunmodulatoren } \\
\text { (Imiquimod, IFN- } \beta \text { ) } \\
\text { Zytostatika (lokal) }\end{array}$ \\
\hline
\end{tabular}

BCC: Basalzellkarzinom, PEC: Plattenepithelkarzinom. 
stellt. Die „Mismatch-Reparatur“, bei deren Fehlfunktion Mikrosatelliteninstabilitäten als Folge von Replikationsfehlern auftreten. Die wichtige Funktion der DNS-Reparatur in dem multifaktoriellen Prozess der Pathogenese von Hauttumoren zeigt sich bei der autosomal rezessiv vererbten Erkrankung Xeroderma pigmentosum, wo die Entwicklung von Hauttumoren wie Basalzellkarzinomen, Plattenepithelkarzinomen und Melanomen mit der defekten Nukleotid-Exzisionsreparatur von UV-induzierten Pyrimidindimeren assoziiert ist [8-10].

Neueste Erkenntnisse wurden auf molekularbiologischer Ebene gewonnen: Apoptose. Wir wissen heute, dass als Ergänzung zu den hauteigenen DNS-Reparaturmechanismen Zellen mit UV-induzierten DNS-Schäden durch Apoptose eliminiert werden können. Eine Fehlfunktion dieses Mechanismus bedingt, dass die DNS-Schädigung nicht zur Apoptose führt, sondern die Zelle maligne entarten kann. An diesem Prozess sind sowohl Tumorsuppressorgene als auch Onkogene beteiligt. Molekularbiologische Veränderungen bei Hauttumoren. Für Basalzell- und Plattenepithelkarzinome der Haut sind Mutationen in unterschiedlichen Genen von Bedeutung: für Plattenepithelkarzinome Mutationen im p53-Gen und für Basalzellkarzinome Mutationen im PTCH-Gen [8]. Eines der am meisten untersuchten Tumorsuppressorgene kodiert für das p53-Protein [11]. Dieses Gen ist in 60 bis $75 \%$ der aktinischen Keratosen, ca. 50\% der Basalzellkarzinome und mehr als $90 \%$ der kutanen Plattenepithelkarzinome mutiert. Darüber hinaus konnte in Plattenepithelkarzinomen der Haut die Inaktivierung des CDKN2A-Tumorsuppressorgens auf dem kurzen Arm von Chromosom 9 (9p21) als eine Progressions-assoziierte Veränderung identifiziert werden. Die relevantesten Mutationen in Onkogenen wurden bei aktinischen Keratosen und Plattenepithelkarzinomen in den RAS-Genen, vornehmlich HRAS und KRAS, nachgewiesen. Beim autosomal dominant vererbten Basalzellnävussyndrom, welches durch das Auftreten zahlloser Basalzellkarzinome gekennzeichnet ist, wurden Mutationen im PTCH-Gen als Ursache identifiziert [12]. Diese Mutationen bewirken nachhaltige Veränderungen in der Signaltransduktion der Zelle.

Diagnostik: Im Vordergrund stehen weiterhin die klinische und histopathologische Untersuchung. Bei pigmentierten Basalzellkarzinomen kann die Auflichtmikroskopie zur differenzialdiagnostischen Klärung beitragen. Möglicherweise stellt die photodynamische Diagnostik bei oberflächlichen Basalzell- und Plattenepithelkarzinomen eine Hilfe in der Abgrenzung zum gesunden Gewebe dar.

Therapie: Das Spektrum der Therapie von epithelialen Hauttumoren hat sich in den letzten 30 Jahren erweitert (Tab. 2). Heute stehen neben den bereits seit Jahrzehnten etablierten Verfahren der operativen Therapie und Strahlentherapie zusätzliche Therapieoptionen wie die photodynamische Therapie, die Lasertherapie oder die topische Anwendung von Immunmodulatoren wie Imiquimod, und in Ausnahmefällen die intra-/peritumorale Injektion von Interferon, zur Verfügung. Die Tumorchirurgie hat heute aufgrund der gewachsenen operativen Kompetenz der Dermatologen die Strahlentherapie weitgehend in den Hintergrund treten lassen. Darüber hinaus waren Dermatologen an der Entwicklung und Etablierung innovativer Anästhesieverfahren maßgeblich beteiligt: Die Tumeszenz-Lokalanästhesie er- laubt auch große tumorchirurgische Eingriffe ohne Vollnarkose durchzuführen.

Evidenz-basierte Untersuchungen, insbesondere zur histographischen Chirurgie, haben zudem zur Optimierung etablierter Therapieverfahren geführt.

\section{Jahre Dermato-Onkologie: Malignes Melanom}

Epidemiologie: Die Inzidenz des malignen Melanoms ist weltweit durch einen starken Anstieg besonders bei der sonnenlichtexponierten weißen Bevölkerung geprägt [13]. In Mitteleuropa werden jährlich 10-12 neue Melanome pro 100000 Einwohner registriert, in Australien 50-60 pro 100 000. Die außerordentliche Zunahme der Erkrankungsfälle in den letzten Jahrzehnten, die zwischen 500-900\% angegeben wird, wird deutlich aus einer Erhebung der Gießener Hautklinik, in deren Einzugsbereich 1975 2,4/100 000, 1984 bereits 11,1/100 000 Einwohner an einem Melanom erkrankten. Aufgrund weltweiter Vorsorge- und Früherkennungskampagnen ist die Mortalitätsrate in den letzten Jahren weitestgehend konstant geblieben.

Klassifikation: Über viele Jahrzehnte erfolgte die Klassifizierung des malignen Melanoms nach der TNM-Klassifikation maligner Tumoren der UICC (Union Internationale Contre le Cancer), die von 1970 bis 1997 in insgesamt 5 Auflagen erschienen ist. Hauptsächliches prognostisches Kriterium war das Tumorstadium, in den Stadien I bis III die Tumordicke (nach Breslow) und die Invasionstiefe (nach Clark). Aufgrund neuerer Auswertungen der Überlebensraten wurde 1994 von Seiten der DDG eine modifizierte Tumorklassifikation vorgeschlagen. Diese kam unter Berücksichtigung einer neuen Stratifizierung der Tumordicken, der Satelliten- und Intransitfiliae zu einer differenzierteren Stadienunterteilung. $2001 \mathrm{kam}$ es in den meisten Kliniken zu einer Neuorientierung im Tumorklassifikationssystem: Die bestehenden Klassifikationen wurden in diesem Jahr in Übereinstimmung mit der WHO, UICC und der Melanomgruppe der EORTC (European Organisation for Research and Treatment of Cancer) durch die Klassifikation der AJCC (American Joint Committee on Cancer) ersetzt (s. Tab. 3, [14]). In diese wesentlich differenziertere Stadieneinteilung haben als neue prognostische Faktoren die Ulzeration des Primärtumors und die nodale Mikrometastasierung Eingang gefunden. Ein entscheidender Unterschied zu den bisherigen Klassifikationssystemen liegt in der Bedeutung des Tumorstadiums für das Überleben des Patienten. Dies wird besonders deutlich bei Ulzeration des Primärtumors: Im Stadium IIB T4a ( $>4 \mathrm{~mm}$ Tumordicke, ohne Ulzeration) N0 M0 ist die 10-JahresÜberlebensrate 53,9\%, während sie im Stadium IIC T4b (> $4 \mathrm{~mm}$ Tumordicke, mit Ulzeration) N0 M0 lediglich 32,3\% beträgt. Das Vorliegen einer Ulzeration bei gleicher Tumordicke bedingt sogar eine schlechtere Prognose als eine nodale Mikro- und Makrometastasierung: In den Stadien IIIA T1a-4a N1a M0 und IIIB T1a-4a N1b M0 betragen die 10-Jahres-Überlebensraten 63\% bzw. $47,7 \%$.

Ätiologie: Im Vergleich zu den Plattenepithelkarzinomen ist die Gewichtung des UV-Lichtes als pathogenetischer Faktor für das maligne Melanom weiterhin nicht gesichert, wie ursprünglich angenommen. In den letzten Jahren wird die These vertreten, 
Tab. 3 Stadieneinteilung nach AJCC (2001)

\begin{tabular}{lllll}
\hline & & & & 10-JÜLZ \\
\hline Stadium 0 & Tis (in situ) & N0 & M0 & ca. $100 \%$ \\
\hline Stadium IA & T1a & N0 & M0 & $87,9 \%$ \\
\hline Stadium IB & T1b/T2a & N0 & M0 & $83,1 / 79,2 \%$ \\
\hline Stadium IIA & T2b/T3a & N0 & M0 & $64,4 / 63,8 \%$ \\
\hline Stadium IIB & T3b/T4a & N0 & M0 & $50,8 / 53,9 \%$ \\
\hline Stadium IIC & T4b & N0 & M0 & $32,3 \%$ \\
\hline Stadium IIIA & T1-4a & N1-2a & M0 & $63,0 / 56,9 \%$ \\
\hline Stadium IIIB & jedes T & N1-2a & M0 & $37,8 / 35,9 \%$ \\
oder & T1-4b & N1-2b/2c & M0 & $47,7 / 39,2 \%$ \\
\hline Stadium IIIC & T1-4b & N1-2b & M0 & $24,4 / 15,0 \%$ \\
\hline Stadium IV & jedes T & N3 & M0 & $18,4 \%$ \\
& jedes T & jedes N & M1a & $15,7 \%$ \\
\hline
\end{tabular}

Tb: + Ulzeration

dass Sonnenbrände in der Kindheit das Risiko der Entstehung von Nävuszelnävi (NZN) steigern. Da eine positive Korrelation zwischen Anzahl von NZN, insbesondere von atypischen NZN und dem Lebenszeitrisiko für die Entstehung von Melanomen besteht, kann dem UV-Licht eine Bedeutung für diesen indirekten Weg in der Pathogenese des Melanoms beigemessen werden.

Epidemiologische Studien konnten allerdings eindeutig eine genetische Disposition für die Entwicklung eines Melanoms nachweisen. So konnten mehrere Studien ein so genanntes Suszeptibilitätsgen für Melanome auf Chromosom 9p21 lokalisieren, welches für ein Zellzyklus-regulierendes Tumorsuppressorgen kodiert ( $16^{\mathrm{INK} 4 \mathrm{a}} / \mathrm{CDKN} 2 \mathrm{~A}$ ). Aktivierende Mutationen wurden in Onkogenen gefunden.

Diagnostik: Erhebliche Fortschritte wurden in der klinischen, mikroskopischen und serologischen Diagnostik des Melanoms erzielt. Das Auge als wichtigstes diagnostisches Instrument des Dermatologen erfuhr eine wesentliche Unterstützung durch die Dermatoskopie und die Videoauflichtmikroskopie, die mittlerweile ein nahezu feinstrukturelles Auflösungsvermögen für die Betrachtung melanozytärer Hautveränderungen ermöglicht und damit einen Beitrag zur Differentialdiagnose dieser Tumoren leistet.

Ein weiteres apparatives Verfahren, welches zu einer außerordentlichen Verbesserung des Tumorstagings führte, stellt die hochauflösende Ultraschalldiagnostik einschließlich farbkodierter Duplexsonographie dar. Während der Einsatz von Ultraschallverfahren in anderen Fachdisziplinen schon seit langem zur Standarddiagnostik gehört, fand sie in der dermatologischen Literatur erstmals 1979 Erwähnung [15]. Mit dieser Methode kann mit hoher Präzision die Tumordicke bestimmt werden. Noch wesentlicher ist der Stellenwert der Methode im Rahmen der primären Ausbreitungsdiagnostik und der Nachsorgeuntersuchungen. Für die Beurteilung des Lymphknotenstatus ist die Sonographie mit einem Auflösungsvermögen von 2-3 mm anderen apparativen Untersuchungen, wie Magnetresonanztomographie, Computertomographie, überlegen.
Ein neuer Weg in der Funktionsdiagnostik maligner Tumoren war die Positronenemissionstomographie (PET), eine bildgebende nicht-invasive Methode u.a. zur Darstellung des Glukosestoffwechsels als Parameter der proliferativen Aktivität eines Tumors [16]. Sie ist als Ganzkörper-PET besonders geeignet für die Darstellung von Lymphknotenmetastasen.

Eine weitere diagnostische Präzisierung des Lymphknotenstatus konnte durch die Wächterlymphknotenbiopsie (SLNB) erreicht werden [17,18]. Mit Hilfe dieser Methode können Mikrometastasen im ersten drainierenden Lymphknoten nachgewiesen werden. Die SLNB stellt eine ergänzende Staging-Untersuchung dar, die eine Aussage über die Prognose erlaubt und das Indikationsspektrum für eine adjuvante Therapie erweitert.

Die Kenntnis der Immunogenität von Melanomzellen und die Charakterisierung zahlreicher Melanomantigene führte zur Etablierung immunhistologischer und serologischer Verfahren. Die Immunphänotypisierung maligner Melanome (u.a. S100 Protein, HMB-45 Antigen) erlaubte eine deutliche Verbesserung der konventionellen histologischen Diagnostik. Die Hoffnung auf einen Tumormarker zur Früherkennung des Melanoms hat sich bislang nicht erfüllt. In der klinischen Routine konnte sich der viel versprechende Ansatz des Nachweises einzelner Melanomzellen im Blut mittels Tyrosinase-RT-PCR nicht durchsetzen [19]. Eine gewisse Bedeutung hat jedoch der Nachweis von S100- $\beta$ Protein, dessen Serumspiegel mit der Prognose von Melanompatienten und einer mit Erfolg durchgeführten Therapie korreliert [20].

Therapie: Die therapeutischen Bemühungen bei Patienten mit malignem Melanom sind gekennzeichnet durch Enttäuschung und Hoffnung zugleich. In der Vergangenheit wurde kein Therapiekonzept unversucht gelassen, diesen Tumor zu heilen, einschließlich anekdotischer Versuche mittels Orchiektomie und Hypophysektomie (Tab.4a; [21]). Den größten Fortschritt für den Patienten mit einer erheblichen Verbesserung der Lebensqualität brachte zum einen die Erkenntnis, dass Sicherheitsabstände von $5 \mathrm{~cm}$ und eine elektive „prophylaktische“ Lymphknotendissektion (ELND) keinen Überlebensvorteil bringen: Die Sicherheitsabstände wurden tumordickenadaptiert auf $1-2 \mathrm{~cm}$ reduziert, die ELND zugunsten der SLNB verlassen. Zum anderen sind neue Möglichkeiten, die Nebenwirkungen systemischer Therapien zu mildern, zu nennen; hier insbesondere die 5- $\mathrm{HT}_{3}$-Rezeptorantagonisten zur Antiemese und die Wachstumsfaktoren zur Gegensteuerung therapielimitierender Leukopenien und Anämien.

Den größten Umbruch erfuhr die adjuvante Therapie (Tab. 4 b). Neben der ELND wurde die adjuvante Strahlentherapie und Chemotherapie verlassen. Von den zahlreichen immuntherapeutischen Ansätzen hat lediglich die Behandlung mit $\alpha$-Interferonen und Vakzinen Bestand.

Die größte Spannweite zwischen Frustration und Faszination spiegelt sich in den vielfältigen Therapieoptionen für Patienten mit metastasiertem Melanom wider (Tab.5) [22-24]. Es hat sich gezeigt, dass sich durch die operative Entfernung von Fernmetastasen in kurativer Intention die Überlebenszeiten bei erhaltener Lebensqualiät verlängern lassen [25]. 
Tab. 4 a Historische und aktuelle kurative Therapiemöglichkeiten des malignen Melanoms

\begin{tabular}{ll}
\hline historisch & aktuell \\
\hline & $\begin{array}{l}\text { Prävention } \\
\text { primär/sekundär }\end{array}$ \\
\hline $\begin{array}{l}\text { operative Therapie } \\
\text { Excision mit } 5 \mathrm{~cm} \text { Sicherheitsabstand } \\
\text { Orchiektomie } \\
\text { Ovarektomie } \\
\text { Amputation }\end{array}$ & $\begin{array}{l}\text { Excision mit stadienadaptiertem } \\
\text { Sicherheitsabstand }\end{array}$ \\
$\begin{array}{l}\text { Strahlentherapie } \\
\text { Chemoimmuntherapie }\end{array}$ & (Strahlentherapie) \\
DNCB & $\varnothing$ \\
\hline
\end{tabular}

Tab. 4b Historische und aktuelle adjuvante Therapiemöglichkeiten des malignen Melanoms

\begin{tabular}{|c|c|}
\hline historisch & aktuell \\
\hline $\begin{array}{l}\text { operativ } \\
\text { Elektive Lymphknotendissektion }\end{array}$ & $\varnothing$ \\
\hline $\begin{array}{l}\text { chemotherapeutisch } \\
\text { Hypertherme Extremitätenperfusion } \\
\text { Monochemotherapie } \\
\text { Polychemotherapie } \\
\text { Kombinierte Chemoimmun-Therapie }\end{array}$ & $\varnothing$ \\
\hline $\begin{array}{l}\text { strahlentherapeutisch } \\
\text { Vor-/Nachbestrahlung } \\
\text { Endolymphatische Radionuklid-Therapie }\end{array}$ & $\varnothing$ \\
\hline $\begin{array}{l}\text { immuntherapeutisch } \\
\text { Levamisol } \\
\text { Transferfaktor } \\
\text { BCG } \\
\text { Corynebacterium parvum } \\
\text { Mistelextrakte } \\
\alpha-, \beta-, \gamma \text {-Interferone } \\
\text { Tumor-/Antigen-Vakzine } \\
\text { Aktive spezifische Immuntherapie (ASI) }\end{array}$ & $\begin{array}{l}\alpha \text {-Interferone } \\
\text { Tumor-/Antigen-Vakzine }\end{array}$ \\
\hline
\end{tabular}

Die Strahlentherapie hat ihren festen Platz im Therapiespektrum des metastasierten Melanoms behalten. Ein besonderes Verfahren stellt die stereotaktische Hochdosis-Einzeit-Photonen-Konvergenzbestrahlung (Radiochirurgie) dar, mit der für bestimmte Patienten ( $<3$ Metastasen, $<3 \mathrm{~cm}$ im Durchmesser) eine große Erleichterung gegenüber der Ganzhirnbestrahlung geschaffen wurde [26].

Die dritte Säule neben operativer und Strahlentherapie war in den vergangenen Jahrzehnten die Chemotherapie. In 1-2\% der Patienten mit einem metastasiertem Melanom kann die Chemotherapie lang anhaltende Tumorremissionen, möglicherweise sogar einen kurativen Erfolg erzielen. Im Rahmen verschiedener Mono- und Polychemotherapiekonzepte wurden und werden Medikamente aus allen Klassen antineoplastisch wirksamer Chemotherapeutika eingesetzt. Seit jeher zeigten die Angaben über die Wirksamkeit für jedes einzelne Medikament und für Kombinationstherapien eine große Schwankungsbreite - meist in Abhängigkeit von der Anzahl behandelter Patienten. So werden für Dacarbazin als Monotherapeutikum Remissionsraten von $14-33 \%$, für eine Polychemotherapie mit Cisplatin, Vinde-
Tab. 5 Aktuelle palliative Therapiemöglichkeiten des malignen Melanoms

operative Therapie
Resektion von Fernmetastasen
Kryotherapie
Lasertherapie
Strahlentherapie
konventionelle Radiotherapie
stereotaktische Radiotherapie
Chemotherapie
Monochemotherapie
Polychemotherapie
kombinierte Zytostatika-/Tamoxifen-Therapie
hypertherme Extremitätenperfusion
regionale Infusion/Perfusion
Immuntherapie
Interleukin-2 (IL-2) intratumoral
Gentherapie/Vakzinierung
Peptid-gepulste dendritische Zellen
Direkte Gentherapie-Ansätze (Gene zur Induktion von Apoptose, z. B.
Antisense-Strategie; Suizid-Gentherapie)
Anti-Angiogenesefaktoren
Chemoimmuntherapie
kombinierte Zytostatika-/Zytokintherapie
hypertherme Extremitätenperfusion

sin, Dacarbazin von 24 - 44\% angegeben. Insgesamt waren die Ansprechraten einer Polychemotherapie höher, jedoch unter gleichzeitiger Inkaufnahme stärkerer Nebenwirkungen. Dies führte zu einem Paradigmenwechsel unter dem Gesichtspunkt „was nützt dem Patienten?“. In erster Linie ist hier nicht die Ansprechrate zu nennen, sondern die Überlebenszeit, und hier insbesondere das Überleben mit erhaltener Lebensqualität. Begriffe wie „TWIST“ (time without symptoms and toxicity) wurden geprägt. Eine wichtige Voraussetzung hierfür war die Linderung von therapiebedingten Nebenwirkungen. Die geänderte Einstellung zur Wertigkeit des Therapieerfolges wird durch neueste Publikationen bestätigt, die auf der Basis randomisierter klinischer Studien zu dem Schluss kommen, dass für die Polychemotherapie kein Vorteil gegenüber einer Monochemotherapie hinsichtlich des Überlebens belegt ist, so dass Dacarbazin seit seiner Einführung vor 30 Jahren weiterhin als Goldstandard angesehen wird. Mit der Entwicklung und dem Einsatz von Immuntherapeutika (Interferon, Interleukin) schien sich zunächst eine vierte Therapiesäule zu etablieren (s. Abb.1), insbesondere im Hinblick auf eine Reduktion der Nebenwirkungen. Die Ansprechraten lagen über $20 \%$. Auch die Hoffnung auf bessere Therapieerfolge durch die Kombination von Zytostatika mit Immuntherapeutika, die so genannte Biochemotherapie, erfüllte sich nicht. Wenngleich ursprünglich Ansprechraten bis zu $73 \%$ berichtet wurden, konnten größere prospektiv randomisierte Studien im Vergleich zur Polychemotherapie kein signifikant verlängertes Überleben nachweisen. Als ein Fortschritt kann die Entwicklung neuer Zytostatika angesehen werden, die bei gleicher Wirksamkeit geringere Nebenwirkungen verursachen bzw. für bestimmte Metastasenlokalisationen, wie Hirnmetastasen, durch eine höhere Liquorgängigkeit zu einer verbesserten lokalen Tumorkontrolle führen (Temozolamide, Fotemustine). Die hohe Anzahl von Patienten, die nicht auf eine Therapie ansprachen, legte den Schluss nahe, dass eine primäre Chemoresistenz der Tumorzellen besteht [27]. Um dem Tumorpatienten möglichst eine für ihn nicht 


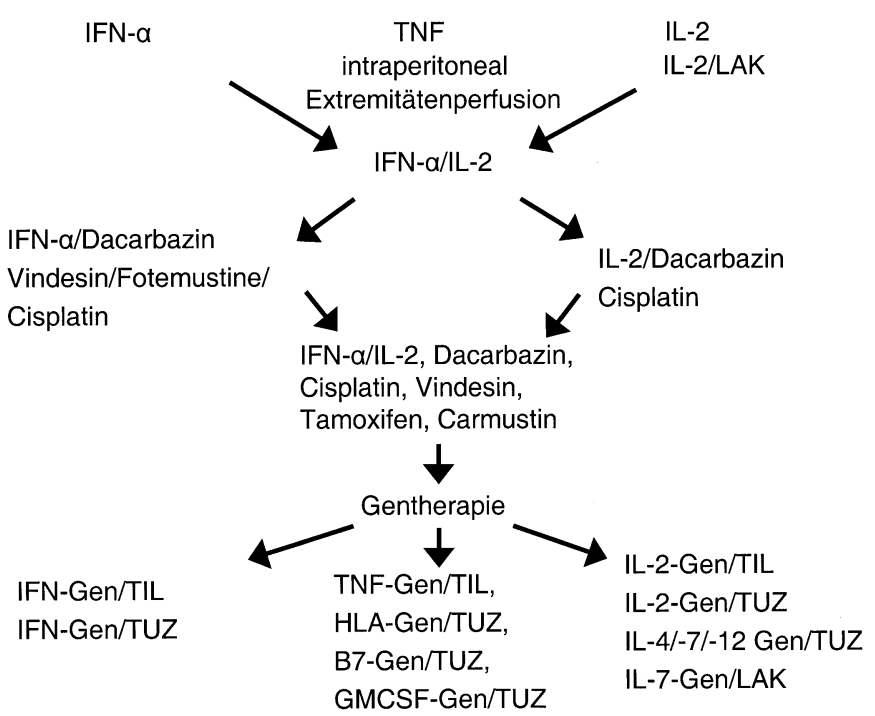

Abb. 1 Zytokine in der Therapie des metastasierten Melanoms.

wirksame Therapie zu ersparen, wurden in der Vergangenheit zahlreiche präklinische Testsysteme entwickelt, die gleich einem Antibiogramm in einem „Onkobiogramm“ vorab die Sensibilität bzw. Resistenz einer Tumorzelle gegenüber einem Zytostatikum zeigen sollen. Derzeit hat keines dieser Systeme Eingang in die klinische Routinediagnostik gefunden. Die neueste Entwicklung stellt die Chemoresistenztestung mittels Biosensor-Chiptechnologie dar.

Das Verständnis um die molekurbiologischen (z. B. Apoptoseresistenz) und immunologischen (z.B. Antigenpräsentation) Vorgänge, die es der Tumorzelle ermöglichen, sich jedweder Behandlung zu entziehen, hat neuartigen Pathogenese-orientierten Therapiestrategien den Weg gebahnt (Abb. 2) [28, 29]. Diese zum Teil innerhalb klinischer Studien überprüften Konzepte zielen auf vier wesentliche Faktoren der Tumorentwicklung ab: Proliferation, Differenzierung, Apoptosehemmung und „Immune escape“-Mechanismen (Abb.3). So wurden unter dem Begriff der
„Targeted“ Therapie Studien initiiert, in denen Kinase-Inhibitoren zur Inaktivierung von Onkogenen mit dem Ziel, die Tumorproliferation einzudämmen, eingesetzt wurden. Ein weiterer Therapieansatz mit dieser Zielrichtung stellt die Entwicklung eines monoklonalen Antikörpers dar, der durch Blockierung eines Wachstumsfaktors der tumorinduzierten Neoangiogenese entgegenwirkt (Bevacizumab). Ferner gelang es mit mRNS blockierenden Nukleotiden (RNS-Antisense-Therapie, Oblimersen) die durch das bcl-2-Protein verursachte Apoptoseinhibition und damit die Chemoresistenz der Tumorzelle aufzuheben. Im letzten Jahrzehnt konnte eine Vielzahl von tumorassoziierten Antigenen identifiziert werden. Damit war die Grundlage für eine gezielte Immuntherapie geschaffen. Wenngleich einige Therapieansätze wieder verlassen wurden (monoklonale Antikörper, Aktive spezifische Immuntherapie - ASI), gelten neue Vakzinationsstrategien als Hoffnungsträger: Die meisten Erfahrungen wurden mit dem Einsatz peptidgepulster Dendritenzellen und epitopbasierter Peptide gesammelt [30].

Nachsorge: Nachsorgekonzepte dienen der Erkennung von Zweitmelanomen und der frühzeitigen Erkennung eines Tumorprogresses. In der Vergangenheit wurden in allen Tumorstadien in kurzen Zeitintervallen umfassende apparative und serologische Untersuchungen durchgeführt. Das Zentralregister Malignes Melanom führte in Tübingen eine prospektive Untersuchung zur Nachsorge bei 2000 Patienten durch. Die Auswertung der Daten zeigte, dass im Vergleich zu den bisherigen Empfehlungen der Umfang der routinemäßig durchgeführten Untersuchungen insbesondere bei Tumoren mit einer Tumordicke $<1 \mathrm{~mm}$ deutlich eingeschränkt werden kann [31]. Dies führt ohne Inkaufnahme eines erhöhten Risikos zu einer erheblichen Entlastung für den Patienten, aber auch für den Kostenträger.

\section{Jahre Dermato-Onkologie: Kutane Lymphome}

Epidemiologie: Die primär kutanen Lymphome stellen mit einer Inzidenz von 0,3-1/100000 Einwohner und Jahr die derzeit zweithäufigste Entität der extranodalen Non-Hodgkin-Lympho-

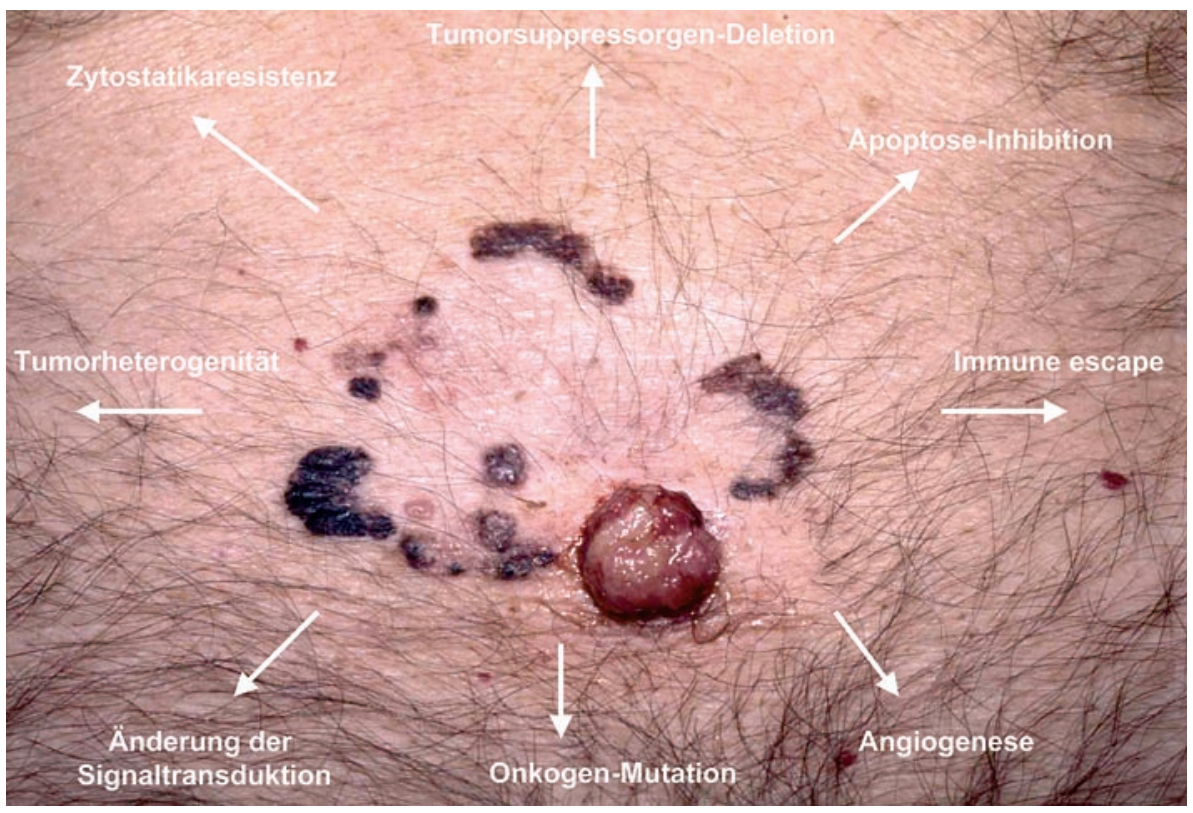

Abb. 2 Molekularbiologische Mechanismen der Tumorprogression beim malignen Melanom. 
$\underset{\text { Therapie }}{\text { Immunologische }}\left\{\begin{array}{l}\text { - Humorale Immunität } \\ - \text { Zelluläre (T-Zell-) Immunität }\end{array}\left\{\begin{array}{l}- \text { Tumorspezifische mAK } \\ - \text { Tumorlysat-Vakzine } \\ - \text { Spezifische Immunisierung } \\ \text { (DC, peptid-gepulst; } \\ \text { Epitop-basierte Peptide; } \\ \text { DNA-Vakzine) }\end{array}\right.\right.$

Gentherapie $\left\{\begin{array}{l}- \text { Transfer von „Suizidgenen“ (HSV-tk) } \\ - \text { Transfer von Tumorsuppressorgenen (p53, p16INK4a) } \\ - \text { Inaktivierung von Onkogenen/onkogenen Signalwegen } \\ \text { (c-myc, ras, Stat3) } \\ - \text { Insertion von Genen, die Zytokine oder kostimulatorische } \\ \text { Moleküle kodieren (IL-2, GM-CSF, IFN's, B7.1) }\end{array}\right.$

"Targeted“ $\quad\left\{\begin{array}{l}\text { Hemmung von: } \\ - \text { Onkogenen (BRAF) } \\ \text { - Apoptose-Inhibitoren (bcl-2) } \\ \text { - Angiogenesefaktoren (VEGF) }\end{array}\left\{\begin{array}{l}\text { - Kinaseinhibitoren BAY 43-9006 } \\ - \text { Antisense RNS Genasense }{ }^{\top M} \\ - \text { Monoklonale AK Bevacizumab }\end{array}\right.\right.$

Abb. 3 Neue Pathogenese-orientierte Therapieansätze bei malignem Melanom.

me (NHL) dar [32 - 33]. Trotz ihres seltenen Auftretens ist in den vergangenen 30 Jahren Entscheidendes geleistet worden, insbesondere für die Klassifikation kutaner Lymphome, die molekulare und immunhistologische Diagnostik und die Etablierung innovativer Therapien.

Klassifikationen: Zu Beginn der 70er-Jahre war das Verständnis der Pathologie kutaner Lymphome ohne Kenntnis immunhistologischer Marker sehr begrenzt. Die Mykosis fungoides (MF) und das Sézary-Syndrom (SS) wurden als Erkrankungen des retikuloendothelialen Systems angesehen. Die für beide Krankheitsbilder charakteristische Lutzner-Zelle wurde zunächst als histiozytäre, nicht als lymphozytäre Zelle klassifiziert. Daher fanden die kutanen Lymphome in ersten umfassenden Lymphom-Klassifikationen keine entsprechende Berücksichtigung. Klinik und Verlauf dieser beiden Entitäten waren hingegen detailliert beschrieben. Unter mehreren alternativen Klassifikationssystemen hat sich 1974 zunächst die so genannte „Kiel-Klassifikation“, die die verschiedenen NHL entsprechend ihrer physiologischen Vorläuferzellen nach zytologischen Kriterien ordnet, etabliert [34]. 1988 erfolgte in einer Überarbeitung der „Kiel-Klassifikation“ die grundsätzliche Unterscheidung von B- und T-Zell-Lymphomen und die Einordnung von MF und SS als T-Zell-Lymphome niedriger Malignität. Erst zehn Jahre später (1997) fanden die kutane Lymphome als eigenständige Entitäten des Hautorgans, deren klinische Verläufe im Gegensatz zu den nodalen Lymphomen einer eigenen Gesetzmäßigkeit folgen und entsprechende Therapiestrategien fordern, in den aktuellen Lymphom-Klassifikationen der EORTC und der WHO Berücksichtigung. Beide Klassifikationen basieren in umfassender Weise auf klinischen, histo- und zytomorphologischen sowie phäno- und genotypischen Merkmalen, wobei die EORTC-Klassifikation Vielfalt und Verlaufsformen der kutanen Lymphome detaillierter beschreibt (Tab.6) $[35,36]$. Es muss als ein besonderer Erfolg für die Dermatologie gewertet werden, dass eine internationale Tumorklassifikation in einem solchen Ausmaß den klinischen Besonderheiten kutaner Lymphome Rechnung trägt.

Ätiologie: Die Ätiologie der kutanen Lymphome bleibt weiterhin ungeklärt. Für die Entstehung primär kutaner Lymphome wird seit Jahrzehnten ein infektiöses Agens als persistierender Antigenstimulus vermutet (Borrelien, Zytomegalievirus). Darüber hinaus sind, abgesehen von der Beobachtung, dass radioaktive Strahlung in sehr hohen Dosen (z.B. Reaktorunfall) zu einem der MF ähnlichen Krankheitsbild führen kann, keine gesicherten Risikofaktoren für die Entstehung kutaner Lymphome bekannt. In den letzten Jahren konnten jedoch entscheidende Erkenntnisse über die Fehlregulation des physiologischen Zelltodes (Apoptose) der entarteten Zellen, die eine wesentliche Rolle für die Progression der Erkrankung spielt, gewonnen werden [32].

Tab. 6 Aktuelle Klassifikation kutaner Lymphome der EORTC und WHO

\begin{tabular}{|c|c|}
\hline EORTC [35] & WHO [36] \\
\hline \multicolumn{2}{|l|}{ Primär kutane T-Zell-Lymphome } \\
\hline $\begin{array}{l}\text { Indolent (Überlebenszeit >10 Jahre) } \\
\text { Mycosis fungoides (MF) } \\
\text { MF mit follikulärer Muzinose } \\
\text { Pagetoide Retikulose } \\
\text { Lymphomatoide Papulose } \\
\text { Großzellige CTCL, CD30+ (anaplastisch, immunoblastisch, pleomorph) }\end{array}$ & $\begin{array}{l}\text { MF } \\
\text { MF Variante } \\
\text { Nicht spezifiziert } \\
\text { CD30+-lymphoproliferative Erkrankung der Haut } \\
\text { Anaplastisches großzelliges Lymphom }\end{array}$ \\
\hline $\begin{array}{l}\text { Aggressiv (Überlebenszeit < } 5 \text { Jahre) } \\
\text { Sézary-Syndrom } \\
\text { Grosszelliges CTCL, CD30- (immunoblastisch, pleomorph) }\end{array}$ & $\begin{array}{l}\text { SS } \\
\text { Peripheres TCL, nicht spezifiziert }\end{array}$ \\
\hline $\begin{array}{l}\text { Provisorisch } \\
\text { Elastolytisches Lymphom (granulomatous slack skin) } \\
\text { Subkutanes Pannikulitis-artiges T-Zell-Lymphom (SPTZL) } \\
\text { Klein- und mittelgroßzellige pleomorphe kutane T-Zell-Lymphome }\end{array}$ & $\begin{array}{l}\text { MF Variante } \\
\text { SPTZL } \\
\text { Peripheres TCL, nicht spezifiziert }\end{array}$ \\
\hline Primär kutane B-Zell-Lymphome & \\
\hline $\begin{array}{l}\text { Indolent (Überlebenszeit > } 10 \text { Jahre) } \\
\text { Keimzentrums-Lymphom (FCCL, follicle center cell lymphoma) } \\
\text { Immunozytom (IZ) bzw. Marginalzonen-Lymphom }\end{array}$ & $\begin{array}{l}\text { Keimzentrums-Lymphom } \\
\text { MZL (niedrig-malignes BCL) vom MALT-Typ }\end{array}$ \\
\hline $\begin{array}{l}\text { Intermediär (Überlebenszeit > } 5 \text { Jahre) } \\
\text { Großzelliges B-Zell Lymphom des Unterschenkels }\end{array}$ & Diffus-großzelliges BCL \\
\hline $\begin{array}{l}\text { Provisorisch } \\
\text { Intravaskuläres großzelliges B-Zell-Lymphom } \\
\text { Plasmozytom }\end{array}$ & $\begin{array}{l}\text { Nicht spezifiziert } \\
\text { Plasmozytom }\end{array}$ \\
\hline
\end{tabular}


Diagnostik: Die Diagnostik kutaner Lymphome wurde in den vergangenen 30 Jahren durch immunologische und molekulargenetische Erkenntnisse entscheidend verbessert. Erstmals gelang 1979 die Immunphänotypisierung von T-Zellen mit monoklonalen Antikörpern (OKT-1, -3, -4), die an Oberflächenproteine dieser Zellen spezifisch binden (CD5, CD3, CD4, s.u.). Diese ist Grundlage der so genannten CD-Klassifikation (cluster of differentiation), die seit 1982 zur Charakterisierung und Klassifikation von Lymphozyten und lymphozytären Neoplasien herangezogen wird. 1983 wurde das Haut-assoziierte lymphatische Gewebe (SALT, skin-associated lymphoid tissue) und 1990 die Existenz eines Faktors (homing factor; cutaneous lymphocyte antigen), der die spezifische Wanderung der T-Lymphozyten in die Haut ermöglicht, beschrieben [37].

Ein weiterer Meilenstein der Diagnostik war 1991 der molekularbiologische Monoklonalitätsnachweis des lymphozytären Infiltrats durch die Entdeckung des Gen-Rearrangements für die Gammakette des T-Zellrezeptors und die Schwerkette des Immunglobulins [38]. Es muss jedoch darauf hingewiesen werden, dass Monoklonalität nicht grundsätzlich gleichzusetzen ist mit Malignität, da auch bei benignen Dermatosen monoklonale Infiltrate nachweisbar sein können.

Therapie: Die Fortschritte in der Behandlung der kutanen Lymphome seit 1974 liegen im Wesentlichen in der Etablierung immuntherapeutischer und moderner phototherapeutischer Verfahren. Daneben haben neue Zytostatika und Retinoide das Armentarium der palliativen Therapie erweitert. Die Therapie der MF vor 30 Jahren bestand in der Anwendung von UV-Licht, lokalen Steroiden und Stickstofflost (Mechlorethamine, nitrogen mustard). Im infiltrativen und Tumorstadium wurden darüber hinaus Röntgenweichstrahlen, schnelle Elektronen und diverse Zytostatika (Cyclophosphamid, Bleomycin, Methotrexat, Chlorambucil mit Prednisolon) appliziert. Die mittlere altersabhängige Überlebenszeit lag bei 2,5 bis 6 Jahren [39]. Heute werden für kutane T-Zell-Lymphome 5-Jahresüberlebensraten von $81 \%$ $100 \%$ bei fehlender Lymphknoten- oder Organbeteiligung berichtet [32]. Wesentliche Säulen der stadienadaptierten Therapieempfehlungen der Deutschen Dermatologischen Gesellschaft (DDG) und des Berufsverbandes Deutscher Dermatologen (BVDD) sind heute Weiterentwicklungen auf dem Gebiet der Lichttherapie (PUVA, 311nm-UVB, extrakorporale Photophorese) sowie die Anwendung von Interferon $\alpha$, selektiven RXR-Antagonisten und liposomal verkapselten Zytostatika. Topische Steroide, Strahlentherapie und Zytostatika sind weiterhin fester Bestandteil des therapeutischen Repertoires [32,40]. Die Überprüfung dieser Therapiestrategien innerhalb von randomisierten multizentrischen Studien führte in den vergangenen 10 Jahren zu einem hohen Qualitätsstandard und zur Aufnahme in die aktuellen interdisziplinären Leitlinien der DKG unter Federführung der ADO [33].

Weitere aktuelle immunologische Therapieansätze wie z. B. Imiquimod, Diphterietoxin-Interleukin-2-Fusionsprotein, Adenovirus-Interferon- $\gamma$ [41] und monoklonale Antikörper (Rituximab, Alemtuzumab) müssen ihre Effektivität in der Behandlung kutaner Lymphome im Rahmen kontrollierter Studien noch unter Beweis stellen.

\section{Schlussbetrachtung}

Wenn man die neuere (30-jährige) Geschichte unseres Faches betrachtet, erkennt man, dass es sich in einem stetigen Umbruch befand und befindet. Die Reputation in der Dermato-Onkologie hat dazu geführt, dass die Eigenständigkeit des Faches und die Einbindung in ein interdisziplinäres Netzwerk auf nationaler und internationaler Ebene Hand in Hand gehen. Fortschritte im Wissen sind der beste Garant für Fortschritte und Erfolge in Diagnostik und Therapie maligner Tumoren. Trotz einer nicht zu leugnenden Kluft zwischen Erfolgen in der Grundlagenforschung und deren Umsetzung in die klinische Anwendung ist der Erkenntniszuwachs über die Zusammenhänge zwischen Tumorprogression und deren genetischen und molekularbiologischen Grundlagen beeindruckend. Bei allem Fortschritt auf molekularer Ebene muss jedoch vor einer Überbewertung dieser Befunde gewarnt werden, da es ohne Berücksichtigung des klinischen Befundes vielfach zu einer Überbehandlung des Patienten kommt. Die beste Therapie ist nach wie vor die Früherkennung von Hauttumoren [42]. Auch wenn es hierfür keinen labortechnischen Tumormarker gibt, so steht uns zur Vorsorge ein stets einsatzbereites und effektives Werkzeug zur Verfügung: die Augen. Voraussetzung für ihre Wirksamkeit ist: Sie müssen sehen gelernt haben.

\section{Literatur}

${ }^{1}$ Trefzer U, Garbe C, Kortmann RD (Redaktion). Leitthemenheft: Melanom. Der Onkologe 2004; 10: 683-753

${ }^{2}$ Garbe C. Entwicklungen von Leitlinien in der Dermatologischen Onkologie. Akt Dermatol 2004; 30: 130-131

${ }^{3}$ Diepgen TL, Mahler V. The epidemiology of skin cancer. Br J Dermatol 2002; 146: $1-6$

${ }^{4}$ Breuninger H, Sebastian G, Garbe C. Deutsche Leitlinie: Plattenepithelkarzinom der Haut. In: Deutsche Krebsgesellschaft e.V: Qualitätssicherung in der Onkologie - Diagnostik und Therapie maligner Erkrankungen. W. Zuckschwerdt-Verlag, 2004

${ }^{5}$ Breuninger H, Sebastian G, Garbe C. Deutsche Leitlinie: Basalzellkarzinom. In: Deutsche Krebsgesellschaft e.V.: Qualitätssicherung in der Onkologie - Diagnostik und Therapie maligner Erkrankungen. W. Zuckschwerdt-Verlag, 2004

${ }^{6}$ Reichrath J, Tilgen W. Hautkrebs und Sonneneinstrahlung. FORUM DKG 2003; 6/03: $36-40$

${ }^{7}$ Altmeyer P, Hoffmann K, Stücker M (eds.). Skin Cancer and UV-Radiation. Berlin: Springer Verlag, 1997

${ }^{8}$ Cleaver JE, Crowley E. UV-damage, DNA repair and skin carcinogenesis. Front Biosci 2002; 7: 1024-1043

9 Rass K. UV damage and DNA repair in basal cell and squamous cell carcinomas. In: Reichrath J (ed). Molecular Mechanisms of Basal Cell and Squamous Cell Carcinomas. Georgetown, TX, USA: Landes Bioscience, in press

${ }^{10}$ Thielmann HW, Popanda O, Edler L, Jung EG. Clinical symptoms and DNA repair characteristics of xeroderma pigmentosum patients from Germany. Cancer Res 1991; 51: 3456 - 3470

${ }^{11}$ Ananthaswamy HN. P53 protein and cancerogenesis of basal cell and squamous cell carcinomas. In: Reichrath J (ed.). Molecular Mechanisms of Basal Cell and Squamous Cell Carcinomas. Georgetown, TX, USA: Landes Bioscience, in press

12 Reifenberger J. Hedgehog/patched/smoothened/GLI signalling pathway and cancerogenesis of basal cell and squamous cell carcinomas. In: Reichrath J (ed.). Molecular Mechanisms of Basal Cell and Squamous Cell Carcinomas. Georgetown, TX, USA: Landes Bioscience, in press

${ }^{13}$ Garbe C, Hauschild A, Volkenandt M et al. Deutsche Leitlinie: Malignes Melanom. In: Deutsche Krebsgesellschaft e.V.: Qualitätssicherung in der Onkologie - Diagnostik und Therapie maligner Erkrankungen. W. Zuckschwerdt-Verlag, 2004 
${ }^{14}$ Balch CM, Buzaid AC, Soong SJ et al. Final version of the American Joint Committee on Cancer staging system for cutaneous melanoma. J Clin Oncol 2001; 19: 3635-3648

${ }^{15}$ Alexander H, Miller DL. Determining thickness with pulsed ultrasound. J Invest Dermatol 1979; 72: 17-19

${ }^{16}$ Tilgen W, Strauss LG, Metz R et al. Die Positronenemissionstomographie (PET): Eine neue Methode zur Funktionsdiagnostik und Therapieplanung bei Patienten mit malignem Melanom. In: Waclawiczek HW et al (Hrsg.). Das Maligne Melanom. Derzeitiger Stand in Diagnose und Therapie. Berlin: Springer Verlag, 1991: 80-87

17 Morton DL, Wen DR, Wong JH et al. Technical details of lymphatic mapping for early stage melanoma. Arch Surg 1992; 127: 392 - 399

18 Chao C, McMasters KM. Update on the use of sentinel node biopsy in patients with melanoma: who and how. Curr Opin Oncol 2002; 14: $217-220$

${ }^{19}$ Gläser R, Rass K, Seiter S, Hauschild A, Christophers E, Tilgen W. Detection of circulating melanoma cells by specific amplification of tyrosinase cDNA can not serve as a tumor marker in melanoma patients: a clinical two-center study. J Clin Oncol 1997; 15: 2818-2825

${ }^{20}$ Hauschild A, Engel G, Brenner W et al. Predictive value of serum S100B for monitoring patients with metastatic melanoma during chemotherapy and/or immunotherapy. Br J Dermatol 1999; 140: 1065 - 1071

${ }^{21}$ Tilgen W. Malignant melanoma: Current therapeutic concepts. Onkologie 1995; 18: $534-547$

22 Eggermont AMM. European approach to the treatment of malignant melanoma. Curr Opin Oncol 2002; 14: 205-211

${ }^{23}$ Keilholz U, Tilgen W, Hohenberger W. Systemische Therapie des metastasierten Melanoms. Ergebnisse randomisierter Studien der letzten zehn Jahre. Dtsch Ärztebl 2003; 100: A 1054-1064

${ }^{24}$ Garbe C, Eigentler TK. Therapie des malignen Melanoms im Stadium der Fernmetastasierung. Hautarzt 2004; 55: 195-213

${ }^{25}$ Wong SL, Coit DG. Role of surgery in patients with stage IV melanoma. Curr Opin Oncol 2004; 16: 155-160

${ }^{26}$ Tarhini AA, Agarwala SS. Management of brain metastases in patients with melanoma. Curr Opin Oncol 2004; 16: 161 - 166

${ }^{27}$ Röckmann H, Schadendorf D. Drug resistance in human melanoma: mechanisms and therapeutic opportunities. Onkologie 2003; 26: $581-587$

${ }^{28}$ Flaherty KT. New molecular targets in melanoma. Curr Opin Oncol 2004; 16: $150-154$

29 Sotomayor MG, Yu H, Antonia S, Sotomayor EM, Pardoll DM. Advances in gene therapy for malignant melanoma. Cancer Control 2002; 9: 3948
${ }^{30}$ Weber JS, Aparicio A. Novel immunologic approaches to the management of malignant melanoma. Curr Opin Oncol 2001; 13: 124-128

${ }^{31}$ Garbe C, Schadendorf D. Malignes Melanom - neue Daten und Konzepte zur Nachsorge. Dtsch Ärztebl 2003; 100: A 1804 - 1808

32 Dippel E, Gellrich S, Klemkle CD, Goerdt S, Sterry W. Kutane T-ZellLymphome. JDDG 2003; 1: 965-980

33 Dummer R, Stadler R, Sterry W. Deutsche Leitlinie: Kutane Lymphome. In: Deutsche Krebsgesellschaft e.V.: Qualitätssicherung in der Onkologie - Diagnostik und Therapie maligner Erkrankungen. W. Zuckschwerdt-Verlag, 2004

${ }^{34}$ Gerard-Marchant R, Hamlin I, Lennert K, Rilke F, Stansfeld A, van Unnik J. Classification of non-Hodgkin's lymphomas. Lancet 1974; 2: 406- 408

${ }^{35}$ Willemze R, Kerl H, Sterry W, Berti E, Cerroni L, Chimenti S, Diaz-Perez JL, Geerts ML, Goos M, Knobler R, Ralfkiaer E, Santucci M, Smith N, Wechsler J, van Vloten WA, Meijer CJ. EORTC classification for primary cutaneous lymphomas: a proposal from the Cutaneous Lymphoma Study Group of the European Organization for Research and Treatment of Cancer. Blood 1997; 90: 354-371

${ }^{36}$ WHO Clinical Advisory Committee Meeting. Classification of Neoplastic Diseases of the Hematopoietic and Lymphoid Systems, Airlie House, Virginia, USA: November 1997. Zitiert in: Stein H, Hiddemann W. Die neue WHO-Klassifikation der malignen Lymphome. Dtsch Ärztebl 1999; 96: A 1054 - 1064

37 Streilein JW. Skin associated lympoid tissues (SALT). Origin and functions. J Invest Dermatol 1983; 80: 12 - 20

38 Trainor KL, Brisco M, Wan J. Gene rearrangement in B- and T-lymphoproliferative disease detected by polymerase chain reaction. Blood 1991; 78: $192-196$

${ }^{39}$ Epstein EH, Levin DL, Croft JD, Lutzner MA. Mycosis fungoides. Survival, prognostic features, response to therapy and autopsy findings. Medicine (Baltimore) 1972; 51: 61-72

40 Beyeler M, Dummer R. Standard- und experimentelle Therapie kutaner T-Zell-Lymphome. Hautarzt 2003; 54: 1177-1184

${ }^{41}$ Dummer R, Hassel JC, Fellenberg F, Eichmüller S, Maier T, Slos P, Acres B, Bleuzen P, Bataille V, Squiban P, Burg G, Urosevic M. Adenovirusmediated intralesional interferon- $\gamma$ gene transfer induces tumor regressions in cutaneous lymphomas. Blood 2004; in press

42 Tilgen W. Die Früherkennung des malignen Melanoms - Ihre prognostische Bedeutung. In: H. Isele (Hrsg.). Onkologie für den Hausarzt, Aktuelle Onkologie 36. W. Zuckschwerdt-Verlag, 1987: 51 - 75 